\title{
Association between industry affiliation and position on cardiovascular risk with rosiglitazone: cross sectional systematic review
}

\author{
Amy T Wang, resident in internal medicine, ${ }^{1,2}$ Christopher P McCoy, chief resident in internal medicine, ${ }^{1}$ \\ Mohammad Hassan Murad, assistant professor of medicine, ${ }^{1,2,3}$ Victor M Montori, professor of medicine $e^{1,2,4}$
}

${ }^{1}$ Department of Internal Medicine, Mayo Clinic, Rochester, MN, USA

${ }^{2}$ Knowledge and Encounter Research Unit, Mayo Clinic Rochester, MN, USA

${ }^{3}$ Division of Preventive, Occupational and Aerospace Medicine, Mayo Clinic, Rochester, MN, USA

${ }^{4}$ Division of Endocrinology, Mayo Clinic, Rochester, MN, USA

Correspondence to: $\mathrm{M} \mathrm{H}$ Murad murad.mohammad@mayo.edu

Cite this as: $B M J$ J 2010;340:C1344 doi:10.1136/bmj.c1344

\section{ABSTRACT}

Objective To explore a possible link between authors' financial conflicts of interest and their position on the association of rosiglitazone with increased risk of myocardial infarction in patients with diabetes.

Data sources On 10 April 2009, we searched Web of Science and Scopus for articles citing and commenting on either of two index publications that contributed key data to the controversy (a meta-analysis of small trials and a subsequent large trial).

Data selection Articles had to comment on rosiglitazone and the risk of myocardial infarction. Guidelines, metaanalyses, reviews, clinical trials, letters, commentaries, and editorials were included.

Data extraction For each article, we sought information about the authors' financial conflicts of interest in the report itself and elsewhere (that is, in all publications within two years of the original publication and online). Two reviewers blinded to the authors' financial relationships independently classified each article as presenting a favourable (that is, rosiglitazone does not increase the risk of myocardial infarction), neutral, or unfavourable view on the risk of myocardial infarction with rosiglitazone and on recommendations on the use of the drug.

Results Of the 202 included articles, 108 (53\%) had a conflict of interest statement. Ninety authors (45\%) had financial conflicts of interest. Authors who had a favourable view of the risk of myocardial infarction with rosiglitazone were more likely to have financial conflicts of interest with manufacturers of antihyperglycaemic agents in general, and with rosiglitazone manufacturers in particular, than authors who had an unfavourable view (rate ratio $3.38,95 \% \mathrm{Cl} 2.26$ to 5.06 and $4.29,2.63$ to 7.02 , respectively). There was likewise a strong association between favourable recommendations on the use of rosiglitazone and financial conflicts of interest (3.36, 1.94 to 5.83). These links persisted when articles rather than authors were used as the unit of analysis $(4.69,2.84$ to 7.72$)$, when the analysis was restricted to opinion articles $(6.29,2.15$ to 18.38$)$ or to articles in which the rosiglitazone controversy was the main focus $(6.50,2.56$ to 16.53$)$, and both in articles published before and after the Food and Drug Administration issued a safety warning for rosiglitazone (3.43, 0.99 to 11.82 and $4.95,2.87$ to 8.53 , respectively).

Conclusions Disclosure rates for financial conflicts of interest were unexpectedly low, and there was a clear and strong link between the orientation of authors' expressed views on the rosiglitazone controversy and their financial conflicts of interest with pharmaceutical companies. Although these findings do not necessarily indicate a causal link between the position taken on the cardiac risk of rosiglitazone in patients with diabetes and the authors' financial conflicts of interest, they underscore the need for further changes in disclosure procedures in order for the scientific record to be trusted.

\section{INTRODUCTION}

The "rosiglitazone controversy" was instigated by a meta-analysis that showed a significant increase in the risk of myocardial infarction associated with the use of the thiazolidinedione drug rosiglitazone in patients with diabetes. ${ }^{1}$ The scientific community responded with a deluge of letters, commentaries, new systematic reviews, and meta-analyses in peer-reviewed journals and in the lay press. These publications presented differing views on the safety of rosiglitazone use-some diametrically opposite.

In 1995, a similar highly contested debate came about after several articles questioned the safety of calcium channel antagonists. In this case, at least part of the controversy appeared to be fuelled or explained by the authors' financial conflicts of interest with the pharmaceutical industry. ${ }^{2}$ Numerous studies have since confirmed an association between conflicts of interest and pro-industry conclusions in articles..$^{3-6}$

In the past decade, research and policy has focused on this association, leading to important progress in policies to manage and encourage disclosure of such financial conflicts of interest. ${ }^{7-9}$ Whether these policies have made any impact on the association between financial conflicts of interest and views expressed in scientific reports is still unknown. ${ }^{10}$ Indeed, the extent to which this association applies to the rosiglitazone controversy is unclear. 
In this systematic review, we aimed to estimate the association between authors' financial conflicts of interest and their published views on the risk of myocardial infarction with rosiglitazone use and recommendations on use of rosiglitazone. We sought also to estimate the prevalence of financial conflicts of interest (defined as the number of studies that have conflicts of interest divided by the total number of studies). Of those articles identified as having financial conflicts of interest, we determined the percentage that appropriately disclosed these relationships.

\section{METHODS}

This review followed a predefined protocol, with sensitivity analyses arranged to answer specific questions that surfaced during study conception. Sensitivity analyses were specified a priori; none of the sensitivity analyses was a post hoc addition.

\section{Article selection}

On 10 April 2009, we used Web of Science and Scopus to search for potentially eligible articles. Eligible articles cited the index publications in the rosiglitazone debate - that is, either the Nissen and Wolski 2007 meta-analysis, ${ }^{1}$ which first highlighted the link between rosiglitazone and cardiovascular events in patients with diabetes, or the interim report of the Rosiglitazone Evaluated for Cardiac Outcomes and Regulation of Glycemia in Diabetes (RECORD) trial, ${ }^{11}$ which was released to respond to the findings of the Nissen and Wolski meta-analysis. To be included, studies had to comment on rosiglitazone and the risk of myocardial infarction. We included original research, reviews, meta-analyses, guidelines, responses, letters, commentaries, and editorials that were not written by the authors of the index publications. Articles written in foreign languages were obtained and translated.

\section{Data extraction}

\section{Data on financial relationships}

One reviewer extracted for all authors of each article data regarding any indication of financial relationships with pharmaceutical companies (for example, conflict or duality of interest statements, employment with a pharmaceutical company, contact information). This data was then blinded (blocked) electronically and irreversibly.

Studies were classified by the nature of the financial conflict of interest and the type of pharmaceutical company. The financial conflicts of interest were defined as: 1) no relationship; 2) a pharmaceutical company funded the article in question; 3 ) the author was employed by a pharmaceutical company; 4) a pharmaceutical company funded research other than that covered in the article in question; or 5) the author was a consultant, advisory board member, speaker, lecturer, or receives travel or honoraria from a pharmaceutical company, or owns stock. The pharmaceutical companies were divided into: 1) rosiglitazone manufacturer (GlaxoSmithKline); 2) pioglitazone manufacturer (Eli Lilly and Takeda); or 3) manufacturer of antihyperglycaemic agents other than rosiglitazone or pioglitazone.
To complete the assessment of financial relations, we searched for disclosure in all publications within two years of the original publication of all contributing authors of each article using Scopus and PubMed. We also conducted a general internet search for conflict of interest using each contributing author's name in combination with various keywords in Google, such as "disclosure," "conflict of interest," "duality of interest," "speaker," "speaker's bureau," "honoraria," and "honorarium."

\section{Grading authors' positions}

Two independent reviewers without financial conflicts of interest graded each article in terms of: 1) the authors' position on the association between rosiglitazone use and the risk of myocardial infarction; and 2) recommendations on the continuation or cessation of rosiglitazone. We used a pre-defined grading system (table 1) based on the system used by Als-Nielsen and colleagues, ${ }^{12}$ and Stelfox and colleagues' classification scale,$^{2}$ to grade authors' enthusiasm for the experimental intervention in the conclusion section of reports of randomised trials.

Authors were classified as having a favourable view on rosiglitozone's safety (that is, rosiglitazone does not increase the risk of myocardial infarction), a neutral view, or an unfavourable view on the risk of myocardial infarction with rosiglitazone and on recommendations on the use of the drug. Strong recommendations calling for the use of pioglitazone, the main competitor drug to rosiglitazone, were also recorded. We did not take into consideration statements regarding the risk of developing congestive heart failure because this disorder is well established as an adverse effect of all available glitazones.

Authors who were identified as neutral on risk of myocardial infarction and favourable or unfavourable on use were classified as favourable or unfavourable, respectively. Likewise, authors that were neutral on use of rosiglitazone and were favourable or unfavourable on risk of myocardial infarction were classified as favourable or unfavourable, respectively. In the case of multiple contributions by a single author, we treated the first author of each eligible paper as the unit of analysis. Authors with neutral articles who had also written favourable or unfavourable articles were considered as having a favourable or an unfavourable opinion, respectively. In the case that an author had both a favourable and an unfavourable opinion, the author was classified as neutral.

During review of the 404 unique items, the two reviewers had 41 disagreements, and after discussion a consensus on each article was reached. A third party was available for final decision if consensus could not be reached. Initial kappa with linear weighting between the two reviewers was 0.83 and 0.85 for the two classification scales respectively.

Rate ratios and 95\% confidence intervals were calculated as a measure of the strength of association between financial conflicts of interest and views on risk of myocardial infarction with rosiglitazone. 
Table 1/Classification scale for author position on rosiglitazone safety and its continued use

\begin{tabular}{lll} 
& \multicolumn{1}{c}{ Risk of myocardial infarction with rosiglitazone } & \multicolumn{1}{c}{ Use of rosiglitazone } \\
\multirow{2}{*}{ Favourable } & $\begin{array}{l}\text { Emphasises that rosiglitazone does not increase the risk } \\
\text { of myocardial infarction }\end{array}$ & $\begin{array}{l}\text { Recommends continued use of } \\
\text { rosiglitazone }\end{array}$ \\
\cline { 2 - 3 } Neutral & $\begin{array}{l}\text { Discredits authors or arguments that emphasise the risk } \\
\text { of myocardial infarction with rosiglitazone }\end{array}$ & $\begin{array}{l}\text { Makes no recommendation } \\
\text { about drug use }\end{array}$ \\
\hline \multirow{2}{*}{$\begin{array}{l}\text { Emphasises that there is insufficient evidence to assess } \\
\text { the risk of myocardial infarction with rosiglitazone }\end{array}$} & $\begin{array}{l}\text { Recommends use of } \\
\text { Unfavourable }\end{array}$ & $\begin{array}{l}\text { Equitably assesses opposing views } \\
\text { of myocardial infarction drugs or }\end{array}$ \\
\cline { 2 - 3 } & $\begin{array}{l}\text { Emphasises that rosiglitazone increases the risk } \\
\text { ofiticises authors who discredit concerns about the risk } \\
\text { of myocardial infarction with rosiglitazone }\end{array}$ & \\
\hline
\end{tabular}

\section{Sensitivity analyses}

We conducted sensitivity analyses to enhance the validity and robustness of our results. On the basis of the approach Stelfox and colleagues used in their investigation of conflicts of interest and the safety of calcium channel blockers, ${ }^{2}$ we combined the two components of the classification scale in table 1 to create one overall rating per article.

In addition, we tested whether using the articles (instead of the authors) as units for analysis would change our conclusions. We also excluded articles written on other subjects that briefly referenced the rosiglitazone controversy. Analysis of only opinion pieces (letters, commentaries, and editorials) was also done. Given that views on the risk of myocardial infarction associated with rosiglitazone might have been affected by the black box safety warning that rosiglitazone could be associated with increased cardiac risk, issued by the Food and Drug Administration on 30 July 2007, ${ }^{13} 14$ we also explored our findings by considering articles published before and after this date.

\section{RESULTS}

\section{Search and retrieval}

We retrieved 847 potentially eligible articles, as judged by their citation link to the index publications; evaluation of their content enabled the exclusion of 634 articles (that is, the articles did not comment on rosiglitazone and the risk of myocardial infarction or use of rosiglitazone). We could not obtain the full text of four articles, which were, therefore, excluded. We also excluded seven articles written by the authors of the index articles. Thus, 202 articles were included in the analyses, including 18 articles that were translated to English before assessment. After accounting for multiple contributions from a single author, 180 unique authors were used for analysis. The article selection process is depicted in the figure. Individual studies are described in the web extra file.

Prevalence and disclosure of financial conflicts of interest Of the 202 eligible studies (10 reported original research, 91 were letters, editorials, or commentaries, and 101 were reviews, meta-analyses, or guidelines), 108 articles $(53 \%)$ included a conflict of interest statement (nine of the $10(90 \%)$ original research articles, 39 of the 91
(43\%) letters, editorials, or commentaries, and 60 of the $101(59 \%)$ reviews, meta-analyses, or guidelines).

A total of 90 (45\%) of the 202 articles were authored by individuals who had financial conflicts of interest. Of the 90 studies with conflicts of interest, 69 (77\%) had a statement disclosing the conflict of interest in the article itself. The other 21 studies with financial conflicts of interest $(23 \%)$ did not disclose these relationships, which were discovered through searching other publications by the same author or the internet. Three (14\%) of these 21 studies published a statement declaring no conflicts of interest. Detailed description of included articles, conflict of interest prevalence, and disclosures are in the web extra file.

\section{Classification of authors' positions}

In terms of the risk of myocardial infarction associated with rosiglitazone, 31 (17\%) of the 180 unique authors offered favourable views, $84(47 \%)$ were neutral, and $65(36 \%)$ had unfavourable views about the safety of rosiglitazone. In terms of recommendations regarding the use of rosiglitazone, 26 authors had favourable views (that is, recommended continued use), 116 were neutral, and 38 held unfavourable opinions.

A large proportion of the authors who offered favourable views about the safety of rosiglitazone had financial conflicts of interest with pharmaceutical companies in general $(29 / 31(94 \%))$, with rosiglitazone manufacturers $(27 / 31(87 \%))$, and with pioglitazone manufacturers $(20 / 31(65 \%))$, compared with those authors expressing unfavourable views (18/65 (28\%), $13 / 65(20 \%)$, and 14/65 (22\%), respectively). Authors who had favourable views on the safety of rosiglitazone were more than three times more likely to have a financial conflict of interest with a pharmaceutical company than were authors who had unfavourable views (rate ratio 3.38, 95\% CI 2.26 to 5.06). There was likewise a strong association between favourable recommendations on the use of rosiglitazone and financial conflicts of interest (rate ratio 3.36, 95\% CI 1.94 to 5.83; table 2).

\section{The use of pioglitazone}

Both the meta-analysis by Nissen and Wolski ${ }^{1}$ and the RECORD trial ${ }^{11}$ looked into the cardiovascular safety of rosiglitazone but not of pioglitazone. The

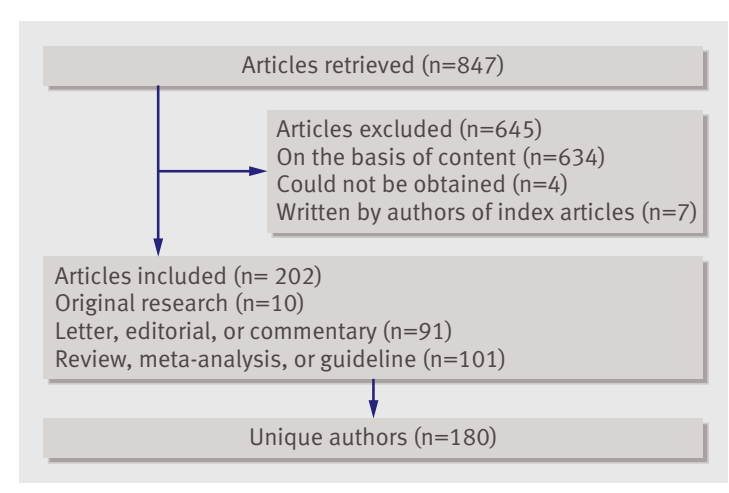

The article selection process 
Table 2 |Author position on rosiglitazone safety and financial conflicts of interest

\begin{tabular}{|c|c|c|c|c|c|c|c|c|}
\hline & \multicolumn{4}{|c|}{ Risk of myocardial infarction with rosiglitazone } & \multicolumn{4}{|c|}{ Use of rosiglitazone } \\
\hline & $\begin{array}{l}\text { Favourable } \\
\qquad(n=31)\end{array}$ & $\begin{array}{c}\text { Neutral } \\
(n=84)\end{array}$ & $\begin{array}{l}\text { Unfavourable } \\
\qquad(n=65)\end{array}$ & Rate ratio $(95 \% \mathrm{Cl})^{*}$ & $\begin{array}{l}\text { Favourable } \\
\quad(n=26)\end{array}$ & $\begin{array}{l}\text { Neutral } \\
(n=116)\end{array}$ & $\begin{array}{l}\text { Unfavourable } \\
\qquad(n=38)\end{array}$ & Rate ratio $(95 \% \mathrm{Cl})^{*}$ \\
\hline Any manufacturer & $29(94)$ & $32(38)$ & $18(28)$ & $3.38(2.26$ to 5.06$)$ & $23(88)$ & $46(40)$ & $10(26)$ & 3.36 (1.94 to 5.83$)$ \\
\hline Rosiglitazone manufacturer† & $27(87)$ & $25(24)$ & $13(20)$ & $4.29(2.63$ to 7.02$)$ & $21(81)$ & $30(26)$ & $9(24)$ & $3.60(2.00$ to 6.48$)$ \\
\hline Pioglitazone manufacturer† & $20(65)$ & $31(30)$ & $14(22)$ & 3.96 (2.45 to 6.39$)$ & $19(73)$ & $30(26)$ & $10(26)$ & 3.28 (1.88 to 5.73$)$ \\
\hline None & $2(6)$ & $52(62)$ & $47(72)$ & - & $3(12)$ & $70(60)$ & $28(74)$ & - \\
\hline
\end{tabular}

Values are numbers (percentages) unless otherwise indicated.

*Comparing favourable versus unfavourable views.

†Categories not mutually exclusive.

PROactive study, however, suggested that pioglitazone may be cardioprotective. ${ }^{15}$

Authors who had favourable views about the use of rosiglitazone were more likely to have a financial conflict of interest with a manufacturer of pioglitazone than were authors with unfavourable views (73\% (19/ 29) $v 26 \%$ (10/44); rate ratio $3.28,95 \%$ CI 1.88 to 5.73$)$. However, 29 articles overall strongly recommended using pioglitazone rather than rosiglitazone, $25(86 \%)$ of which were authored by individuals with financial conflicts of interest with a pioglitazone manufacturer.

\section{Sensitivity analyses}

All sensitivity analyses are presented in part $\mathrm{E}$ of the web extra file. Our results were similar when we performed analyses using Stelfox and colleagues' combined scale ${ }^{2}$ : $42(23 \%)$ authors were classified as having a favourable opinion on the risk of myocardial infarction with rosiglitazone, $70(39 \%)$ were neutral, and $68(38 \%)$ had an unfavourable view. One author had disparate views on the risk of myocardial infarction and its continued use and was thus classified as neutral. When we looked at the link between authors' views on the safety of rosiglitazone and financial conflicts of interest, the results were similar to those in our original analysis. A total of $79 \%$ (33/42) of authors who expressed a favourable view had financial conflicts of interest with the manufacturer of rosiglitazone, compared with $22 \%$ $(15 / 68)$ of authors who expressed an unfavourable opinion (rate ratio $3.65,95 \%$ CI 2.31 to 5.77 ).

When we used each article as a unit of analysis, we found that $35(17 \%)$ were favourable, $95(47 \%)$ were neutral, and $72(36 \%)$ were unfavourable in terms of risk of myocardial infarction with rosiglitazone. In total, $86 \%(30 / 35)$ of favourable articles were associated with a financial conflict of interest with the manufacturer of rosiglitazone, compared with 18\% (13/72) of articles that reported an unfavourable viewpoint (rate ratio $4.69,95 \%$ CI 2.84 to 7.72 ).

When we restricted the analysis to the 105 articles in which the rosiglitazone controversy was the main focus, we found that articles whose authors had favourable views on the risk of myocardial infarction with rosiglitazone were more likely to have financial conflicts of interest with the manufacturer of rosiglitazone than articles expressing unfavourable views (84\% (21/ 25) $v 14 \%$ (7/51); rate ratio $6.00,95 \%$ CI 2.98 to 12.10$)$.
We also found this relation when we undertook an analysis of recommendations on use: $81 \%(13 / 16)$ of articles expressing a favourable recommendation had financial conflicts of interest with the manufacturer of rosiglitazone, compared with 13\% (4/31) of articles expressing an unfavourable recommendation (rate ratio $6.50,95 \%$ CI 2.56 to 16.53$)$.

Results were also consistent when the analysis was restricted to the 92 publications that were a commentary, letter, or editorial: $90 \%$ (19/21) of articles expressing a favourable view on the risk of myocardial infarction with rosiglitazone had financial conflicts of interest with the manufacturer of rosiglitazone, compared with $14 \%(5 / 35)$ of articles expressing an unfavourable view (rate ratio $6.15,95 \%$ CI 2.71 to 13.99 ). Analysis of the authors' recommendations on rosiglitazone use yielded nearly identical numbers $(80 \%$ (12/ 15) $v 13 \%$ (3/23); rate ratio $6.29,95 \%$ CI 2.15 to 18.38$)$.

A total of 27 articles were published before the FDA issued a black box safety warning for rosiglitazone. Of these 27 articles, eight (30\%) were favourable, 10 (37\%) were neutral, and nine (33\%) were unfavourable about rosiglitazone safety. Of the articles in which authors expressed favourable views on rosiglitazone safety, $75 \%$ (6/8) were associated with financial conflicts of interest with rosiglitazone manufacturers compared with $22 \%(2 / 9)$ in which authors had an unfavourable view (rate ratio $3.43,95 \%$ CI 0.99 to 11.82 ). Similar results were obtained for the recommendations on rosiglitazone use for this subset of articles (75\% (3/4) $v 17 \%(1 / 6)$; rate ratio $4.50,95 \%$ CI 0.69 to 29.39$)$.

We then repeated our analysis for articles published after the FDA warning. We found that $89 \%$ (24/27) of articles in which authors presented favourable views were associated with financial conflicts of interest with the rosiglitazone manufacturer compared with $17 \%(11 / 63)$ of articles written by unfavourable authors. Similar results were obtained for the recommendations on rosiglitazone use for this subset of articles $(84 \%(21 / 25)$ v 24\% (9/38); rate ratio 3.75, $95 \% \mathrm{CI}$ 2.10 to 6.72$)$.

\section{DISCUSSION}

Our results show a strong association between authors with favourable views on the safety of rosiglitazone and financial conflicts of interest the authors had with the manufacturer of rosiglitazone, pioglitazone, and with 
any manufacturer of antihyperglycaemic agents. Authors who were unfavourable on the issue of rosiglitazone safety were largely free of identifiable financial conflicts of interest. Sensitivity analyses demonstrated that these links persisted when articles rather than authors were used as the unit of analysis, when the analysis was restricted to opinion articles or to articles in which the rosiglitazone controversy was the main focus, and both in articles published before and after the FDA issued a safety warning.

Owing to increased demands for transparency, most major journals have a disclosure policy that includes not only original articles, but also letters, editorials, and other article types. With requirements for disclosure of conflicts of interest seemingly ubiquitous in medical journals, it was surprising to see that only half of the articles we analysed had competing interests statements. Our findings underscore the need for accountability by both authors and journals. The rate of disclosure we found represents remarkable progress over the past decade, however, because in 1998 Stelfox and colleagues reported that only $3 \%$ of studies had a conflict of interest statement. ${ }^{2}$ Given the potential influence of financial conflicts of interest on the views expressed in articles, the low rate of disclosure by authors of editorials and commentaries is disappointing.

\section{Strengths and limitations of the study}

We used a comprehensive search strategy to identify articles for our analysis, and reviewed articles in duplicate. This approach was associated with high interobserver agreement regarding the classifications of authors' financial conflicts of interest and expressed views and recommendations. We also blinded the reviewers who classified authors' positions relative to the source that funded the research in order to reduce the risk of bias. Multiple sensitivity analyses were planned and conducted; the similarity of their results to those of the primary analyses demonstrates the robustness of our findings.

The inferences presented in this study are limited, however, by the observational and descriptive nature of this evidence. Our analysis was unable to evaluate the effect of the strength of financial associations (that is, assign monetary magnitude to the relationships). Furthermore, the findings rely exclusively on the pertinence and reproducibility of the classifications of the financial conflicts of interest and the positions articulated by the authors.

We were unable to clarify the effect of financial conflicts of interest with the manufacturers of rosiglitazone's direct competitor, pioglitazone, because the majority $(82 \%)$ of authors who had financial conflicts of interest with pioglitazone manufacturers also had financial conflicts of interest with the maker of rosiglitazone. This link may explain the apparently counterintuitive finding that a large proportion of authors with a favourable view about rosiglitazone's safety also had financial conflicts of interest with manufacturers of pioglitazone. This again underscores the complexity of financial conflicts of interest, the extent to which they affect key opinion leaders, and the shortcomings of the current approach to disclosure.

Only $53 \%$ of the articles had conflict of interest statements, thus we relied on exhaustive PubMed, Scopus, and Google searches to identify unreported financial conflicts of interest. Given that no formal database exists for reporting conflicts of interest, our findings may very well underestimate the prevalence of conflicts of interest among authors weighing in on rosiglitazone's safety. We also could not assess the extent to which the observed association reflects ghostwriting in publications related to rosiglitazone. ${ }^{16}$

We found many articles that cited the index studies but did not reference the controversy surrounding the risk of myocardial infarction with rosiglitazone; thus, a number of articles that could have been classified as neutral were likely excluded. We might have also missed articles that refer to the effect of rosiglitazone on the risk of myocardial infarction, either favourably or unfavourably, without directly citing the index articles. In addition, original research often has a lag time associated with publication that may lead to bias in the sensitivity analyses of articles published before the FDA's black box warning label and those published after. Of note, only 10 original research studies were included in these analyses, which limits the potential effect of this bias.

Other limitations may be caused by the characteristics of authors. Authors who have favourable views on rosiglitazone may be more likely to form relationships with manufacturers of glitazones. Cardiologists are less likely to have an association with glitazone manufacturers than are endocrinologists, although cardiologists might be more concerned and vocal about the risk of myocardial infarction with rosiglitazone than are endocrinologists (who may be more concerned about rosiglitazone's effects on glycaemic control).

\section{Conclusions}

The disclosure rates of financial conflicts of interest were unexpectedly low, and there was a clear and strong link between the orientation of authors' expressed views on the rosiglitazone controversy and the existence of financial conflicts of interest with pharmaceutical companies. These findings, while not necessarily causal, underscore the need for further progress in reporting in order for the scientific record to be trusted.

In the heat of the rosiglitazone controversy, patients and clinicians alike were exposed to many arguments on both sides of the debate. How could interpretation of the same evidence result in disparate and impassioned positions? We aimed to determine whether financial conflicts of interest with pharmaceutical manufacturers could be fuelling this fire. From our findings, it appears that the answer is yes. We realise that key opinion leaders who report competing financial interests may not necessarily be biased by these financial relationships, but our findings indicate a strong association that users of the scientific literature should recognise. 
What is already known on this topic

Recent demands for increased transparency have led to stricter and more prevalent policies on disclosures of conflicts of interest

Over the past decade, various studies have shown an association in published articles between conflicts of interest and proindustry conclusions

What this study adds

Despite the ubiquity of requirements for reporting conflicts of interest, the presence of conflict of interest statements in the articles we assessed was low (53\%)

Authors who had favourable views on the safety of rosiglitazone were more likely to have a financial conflict of interest with a pharmaceutical company than were authors who had unfavourable views

Our findings serve as a call to action for an ongoing conversation on how best to foster objectivity in readers and writers of the scientific literature

We need to further the conversation on this topic to stimulate novel ideas that can address the issue of myocardial infarction with rosiglitazone. One method would be controlling for investigator bias in research studies as routinely as we control for other biases, with one report proposing conducting standard conflict of interest analyses to see if reported financial ties are predictive of conclusion. ${ }^{17}$

Contributors: ATW, MHM, and VMM conceived and designed the study. ATW and CPM reviewed articles and extracted data. ATW and MHM analysed the data. ATW, MHM, and VMM wrote the paper. All authors had full access to the data in the study, can take responsibility for the integrity of the data and the accuracy of the data analysis, and edited and approved the final version. VMM is the guarantor.

Funding: No funding was required for this study.

Competing interests: All authors have completed the Unified Competing Interest form at www.icmje.org/coi_disclosure.pdf (available on request from the corresponding author) and declare (1) No financial support for the submitted work from anyone other than their employer; (2) No financial relationships with commercial entities that might have an interest in the submitted work; (3) No spouses, partners, or children with relationships with commercial entities that might have an interest in the submitted work; (4) No non-financial interests that may be relevant to the submitted work.

Ethical approval: Not required.

Data sharing: All data is in the web extra file. No additional data is available.

1 Nissen SE, Wolski K. Effect of rosiglitazone on the risk of myocardia infarction and death from cardiovascular causes. N Engl J Med 2007;356:2457-71.
2 Stelfox HT, Chua G, O’Rourke K, Detsky AS. Conflict of interest in the debate over calcium-channel antagonists. N Engl J Med 1998;338:101-6.

3 Bekelman JE, Li Y, Gross CP. Scope and impact of financial conflicts of interest in biomedical research: a systematic review. JAMA 2003;289:454-65.

4 Bhandari M, Busse JW, Jackowski D, Montori VM, Schunemann H, Sprague S, et al. Association between industry funding and statistically significant pro-industry findings in medical and surgical randomized trials. CMA/ 2004;170:477-80.

5 Jørgensen A, Hilden J, Gøtzsche P. Cochrane reviews compared with industry supported meta-analyses and other meta-analyses of the same drugs: systematic review. BMJ 2006;333:782.

6 Lexchin J, Bero LA, Djulbegovic B, Clark O. Pharmaceutical industry sponsorship and research outcome and quality: systematic review. BMJ 2003;326:1167-70.

7 Brennan TA, Mello MM. Sunshine laws and the pharmaceutical industry. JAMA 2007;297:1255-7.

8 ICGME (International Committee of Medical Journal Editors). Uniform requirements for manuscripts submitted to biomedical journals: writing and editing for biomedical publication. 2008. www.icmje. org/2008_urm.pdf.

9 Ross JS, Lackner JE, Lurie P, Gross CP, Wolfe S, Krumholz HM. Pharmaceutical company payments to physicians: early experiences with disclosure laws in Vermont and Minnesota. JAMA 2007;297:1216-23.

10 Spelsberg A, Martiny A, Schoenhoefer PS. Is disclosure of potential conflicts of interest in medicine and public health sufficient to increase transparency and decrease corruption? J Epidemiol Community Health 2009;63:603-5.

11 Home PD, Pocock SJ, Beck-Nielsen H, Gomis R, Hanefeld M, Jones NP, et al. Rosiglitazone evaluated for cardiovascular outcomes —an interim analysis. N Engl J Med 2007;357:28-38.

12 Als-Nielsen B, Chen WD, Gluud C, Kjaergard LL. Association of funding and conclusions in randomized drug trials-a reflection of treatment effect or adverse events? JAMA 2003;290:921-8.

13 Food and Drug Administration. FDA adds boxed warning for heartrelated risks to anti-diabetes drug Avandia. Agency says drug to remain on market, while safety assessment continues. 2007. www. fda.gov/NewsEvents/Newsroom/PressAnnouncements/2007/ ucm109026.htm.

14 Starner Cl, Schafer JA, Heaton AH, Gleason PP. Rosiglitazone and pioglitazone utilization from January 2007 through May 2008 associated with five risk-warning events. J Manag Care Pharm 2008;14:523-31.

15 Dormandy JA, Charbonnel B, Eckland DJ, Erdmann E, Massi-Benedetti M, Moules IK, et al. Secondary prevention of macrovascular events in patients with type 2 diabetes in the PROactive Study (PROspective pioglitAzone Clinical Trial In macroVascular Events): a randomised controlled trial. Lancet 2005;366:1279-89.

16 Ross JS, Hill KP, Egilman DS, Krumholz HM. Guest authorship and ghostwriting in publications related to rofecoxib: a case study of industry documents from rofecoxib litigation. JAMA 2008;299:1800-12.

17 Greenland S. Accounting for uncertainty about investigator bias: disclosure is informative. J Epidemiol Community Health 2009;63:593-8.

Accepted: 21 December 2009 